\title{
Controlling and Mapping Interfacial Stress Transfer in Fragmented Hybrid Carbon Fibre-Carbon Nanotube Composites
}

\author{
S.-Y. Jin ${ }^{1}$, R.J. Young ${ }^{1}$, S.J. Eichhorn ${ }^{* 2}$ \\ 1. Materials Science Centre, School of Materials, University of Manchester, Grosvenor \\ Street, Manchester, M13 9PL, UK. \\ 2. College of Engineering, Maths \& Physical Sciences, Physics Building, University of \\ Exeter, Stocker Road, Exeter, Devon, EX4 4QL, UK.
}

\begin{abstract}
Raman spectroscopy was used to map the stress transfer at the interface between high and low modulus carbon fibres in model composites when undergoing fragmentation. Both fibre surfaces were coated with two types of single wall carbon nanotubes (HiPCO and carboxylated nanotubes) in order to enhance the interfacial shear strength with an epoxy resin. For the low modulus carbon fibre this coating also enabled stress mapping at the interface. In both cases single fibres embedded in a dumbbell shaped model composite were deformed to cause fragmentation. When no further fragmentation took place the critical fibre length was calculated and converted to interfacial shear stress using classical Kelly-Tyson theory. These values were compared to data obtained using a Raman spectroscopic approach where the rate of change of stress with respect to distance along the fibre was measured directly. These data were then shown to fit a shear lag model. Two forms of single-wall carbon nanotubes were compared; namely unmodified and $\mathrm{COOH}$ modified. It was shown that only the $\mathrm{COOH}$ modified single wall carbon nanotubes increase the maximum interfacial shear stress significantly.

\footnotetext{
${ }^{*}$ Corresponding Author. Tel. : +44 0139272 5515; fax: +44 01392264 111. E-mail address: s.j.eichhorn@exeter.ac.uk (S.J. Eichhorn)
} 
Evidence of matrix yielding at the fibre ends is also presented and the possibility of the enhancement of the shear yield stress of the resin by the presence of the nanotubes is also discussed.

Keywords: (A) Carbon fibres; (A) Carbon nanotubes; (B) Interface; (D) Raman spectroscopy

\section{Introduction}

There has been a surge of interest in carbon fibres in recent times due to their widening use in applications ranging from aerospace, automotive, wind/tidal, sports goods and infrastructure sectors. A number of carbon fibres with different mechanical properties are currently commercially available, ranging from low to high modulus and strength. Their performance in composite materials is predicated on the properties of the interface between the fibre and a resin, and also interactions between fibres themselves.

A number of techniques have been developed to assess the strength of the interface between brittle fibres (e.g. carbon) and resin matrix materials. One of the best known approaches is to use fragmentation. This method, introduced by Kelly and Tyson [1] embeds a free length of fibre into a block of resin which is then deformed in tension. During tensile deformation stress is transferred to the fibre progressively and it fragments it into smaller and smaller lengths shorter or equal to the critical length $l_{\mathrm{c}}[1$, 2]. Typically the test is conducted using a ductile resin, which is often not really representative of a structural composite, but does allow for a complete "saturation" of fragmentation events. When no further fragments are produced during deformation the 
process is said to have reached saturation. At this point typically the lengths of fragments are measured and the interfacial shear stress $\left(\tau_{\mathrm{i}}\right)$ can be determined using a force balance argument leading to the equation [2]

$$
\tau_{\mathrm{i}}=\frac{\sigma_{\mathrm{f}} d}{2 l_{\mathrm{c}}}
$$

where $\sigma_{\mathrm{f}}$ is the fibre strength, $d$ is the fibre diameter and $l_{\mathrm{c}}$ is the critical length, which is assumed to be a single value from the mean of the distribution of fibre lengths at saturation. The critical length $l_{\mathrm{c}}$ is not the same as the arithmetic fragment length, however. A fibre with a length $>l_{\mathrm{c}}$ can break into two lengths $<l_{\mathrm{c}}$. The distribution of fragment lengths $\left(l_{\mathrm{f}}\right)$ is typically therefore in the range $l_{\mathrm{c}} / 2 \leq l_{\mathrm{f}} \leq l_{\mathrm{c}}$ with a mean of $3 l_{\mathrm{c}} / 4$. So,

$$
l_{\mathrm{c}}=4 / 3\left(l_{\mathrm{f}}\right)
$$

and equation (1) can be re-written as

$$
\tau_{\mathrm{i}}=\frac{3}{8} \frac{\sigma_{\mathrm{f}}^{*} d}{l_{\mathrm{f}}}
$$

where $\sigma_{f}^{*}$ is the tensile strength of a fibre at the critical length. 
There are a number of issues with this approach to interfacial shear stress calculations. One of the fundamental assumptions of the fragmentation approach is that there is a constant shear stress at the interface. Plastic deformation events such as debonding and matrix yielding can also take place which can affect the measurement of the true fragment length related to the shear stress at the interface. The fibre length distribution is also very dependent on the fibre strength distribution, which in itself is not a single value, varying along the length of a single fragment due to the presence of statistical flaws. As the process of fragmentation progresses smaller fibre lengths are effectively stronger and so fibre strength is a constantly changing parameter [2]. There is no way of tracking this adequately in a standard fragmentation test. In addition both the shear stress along the interface and the fibre diameter are assumed to be constant; both these parameters can change significantly. For these reasons various approaches have been adopted to obtain effective interfacial shear stresses using statistical data of both fragmentation lengths and strengths [3]. An approach of measuring local stress states directly in a free fibre during fragmentation using Raman spectroscopy has been previously reported [4-6]. This approach enables a point-to-point mapping of the interfacial shear stress in a model composite, and the identification of plastic deformation events such as debonding and matrix yielding.

In the present work we build upon a previous paper from our group where we showed that local stress states can be obtained from low modulus carbon fibres (LMCF) using a coating of single-wall carbon nanotubes (SWNT) on their surface [7]. We show that the maximum interfacial shear stresses obtained using Raman spectroscopy are higher than those predicted using standard fragmentation analysis. Furthermore it is shown that the 
presence of nanotubes increases the interfacial shear stress significantly for both low(LMCFs) and high-modulus carbon fibres (HMCFs).

\section{Experimental Methods}

\section{$2.1 \quad$ Materials}

Two commercial grades of carbon fibre were used for this study; namely a high modulus carbon fibre (HMCF; code M46J) provided by Toray and a low modulus fibre (LMCF; name Tenax-J) by Toho-Tenax. Each fibre was used as-received and had no pre-treatment of its surface. Two types of single wall carbon nanotubes (SWNTs) were coated on the fibres. The first type - HiPCO SWNTs - are produced using a high pressure and a carbon monoxide process. The HiPCO SWNTs were supplied by the American company NanoIntegris. Carboxylic acid treated $(\mathrm{COOH})$ single wall carbon nanotubes (SWNTs) were provided by Sigma Aldrich (Sigma Aldrich, Dorset, UK). The technical data sheet for both these forms of SWNTs states that they have diameters in the range $1.3-1.5 \mathrm{~nm}$, lengths in the range 500-1500 nm and that they contain $<15$ wt.\% metals. The epoxy resin used as a matrix material for the model composites was provided by Vantico, Polymer Specialties, UK. The formulation is a two part curing system of a resin (LY5052) containing 34-42\% 1,4-butanediol diglycidyl ether and 6070\% epoxy phenol-novolac resin and a hardener (HY5052) comprised 35\% isophorone diamine, 50-60\% 2,2-dimethyl-4,4-methylene bis(cyclohexylamine) and 1-5\% 2,4,6tris(dimethylaminomethyl) phenol. The silane coupling agent used in this study was 3aminopropyl-triethoxysilane which is supplied by Avocado Research Chemicals, UK. A release agent named Ambersil Formula 10 was used, which is a dry film, non-silicone mould release agent and was provided by Ambersil House, UK. 


\subsection{Model Composite Preparation}

Coating of the fibres and their mechanical testing and properties have been reported in a previous publication [7]. In addition to hot curing of the coating, a cold cure was also carried out for comparison. To produce model composite specimens from the coated fibres for the fragmentation experiments, one third of an epoxy resin/hardener mix (LY5052/HY5052) was poured into a dumbbell shaped mould into which release agent was sprayed. An $18 \mathrm{~mm}$ length of carbon fibre was then placed into the central region of the dumbbell shape, parallel to the tensile axis, after 1 hour of curing when the resin was partially solid. After this the remaining two thirds of the resin was poured into the mould and the system was hot cured at a temperature of $120{ }^{\circ} \mathrm{C}$ for 2 hours. Finally the samples were removed from the mould and the edges were polished to eradicate surface flaws. The final thickness of the samples was $\sim 2 \mathrm{~mm}$. A strain gauge was carefully attached to the sample surface using cyanoacrylate adhesive. Two wires were then soldered onto the strain gauge and connected to a voltmeter to record the strain. To apply tensile deformation to the samples a cured epoxy dumbbell specimen was fixed on a MINIMAT mechanical testing rig.

\subsection{Raman spectroscopy of Single Fibres and Model Composites}

Composite samples were deformed in steps of $0.3 \%$ strain. Strain was recorded in the specimen using the attached gauge as described in Section 2.2. After each strain increment a Raman spectrum was recorded along the free length of the fibre using a Renishaw System 1000 spectrometer and a He-Ne laser $(\lambda=632 \mathrm{~nm})$ focussed to a spot size of 1-2 $\mu \mathrm{m}$ using a microscope with a $\times 50$ objective lens. A range of exposure times were used for the samples. For single fibres in free air, without the presence of 
nanotubes, an exposure time of $20 \mathrm{~s}$ with 2 accumulations was adequate to obtain clear spectra. This was reduced to $10 \mathrm{~s}$ with the presence of nanotubes since a strong resonance effect is obtained from the latter. In order to obtain spectra from the composite specimens an exposure time of $20 \mathrm{~s}$ was used. Following collection the data were analysed using GRAMS-32 software to obtain the peak positions by fitting with a mixed Gaussian-Lorentzian function. Single fibres were deformed in air on a paper testing card, as has been previously described [7]. Strain increments of $0.1 \%$ were used to deform the single fibre samples, and at each increment a Raman spectrum was recorded. Point-to-point collection of Raman spectra along an intact fibre length, and along fragments during model composites' testing, were recorded at increasing static strain increments of $0.3 \%$ up to a maximum of $2.3 \%$.

\section{Results and Discussion}

\subsection{Raman spectroscopy of Single Fibres and Model Composites}

Typical Raman spectra obtained from both HMCFs and LMCFs and SWNTs $(\mathrm{COOH}$ modified) are shown in Figure 1. The Raman spectrum of carbon fibres is typified by the presence of up to 3 distinct bands; namely the D-band, the G-band and the 2D-band. All three bands are present in the spectra obtained from HMCFs (Fig. 1a) and SWNTs (Fig. 1c). Only two bands (D- and G-bands) are present in the spectrum for LMCFs (Fig. 1b). The 2D band, located at $\sim 2660 \mathrm{~cm}^{-1}$ is thought to be an overtone of the Dband, located at $\sim 1340 \mathrm{~cm}^{-1}$, which is related to the breathing mode of the six-fold aromatic ring near the graphite basal edge [8]. The G-band is thought to be an in-plane bond stretching mode of hybridised $\mathrm{sp}^{2} \mathrm{C}$ atoms [8]. The absence of a 2D-band in the Raman spectrum obtained for a LMCF (Fig. 1b) makes it difficult to high precision 
perform stress analysis, although it can be carried out using the G-band [9]. As has been noted previously [7], when SWNTs are coated on the fibre surface it is possible then to perform such analysis with precision. Rotating the samples under a polarised laser (polarisation applied parallel to the axis of the fibre and with the analyser in collecting only parallel scattered light - VV) reveals that the SWNTs are preferentially oriented parallel to the fibre axis. An example of such preferential orientation is shown in Fig. 1d where it can be seen that the intensity of the $2 \mathrm{D}$ band is maximum parallel to the axis of the fibre $\left(0^{\circ}\right.$ and $\left.180^{\circ}\right)$, and falls to a minimum when the axis is rotated transverse to the polarisation direction $\left(90^{\circ}\right)$. The nanotubes were applied to the fibres as a suspension in a silane solution [7]. It is likely that they became aligned to the surface of the fibres through drawing from the solution and during shrinkage of the silane coating upon evaporation of the solvent.

When the fibres were deformed in tension as single filaments in air, or when embedded in a model composite the $2 \mathrm{D}$ band was found to shift towards a lower wavenumber position. An example of a typical shift in the band position is shown in Figure $2 \mathrm{a}$ and is thought to be due to direct stressing of the $\mathrm{C}-\mathrm{C}$ bonds within the graphitic structure of the fibres. The effect has been reported extensively both for carbon fibres and SWNTs e.g. refs [9-12].Detailed shifts in the 2D peak positions with tensile deformation for SWNTs coated on the surface of a LMCF (in this case COOH modified SWNTs) are shown in Figure $2 \mathrm{~b}$. In this figure data for both hot and cold cured coatings are reported for comparison. It is clear that the application of hot curing to the coating appears to increase the shift rate in the $2 \mathrm{D}$ band position with respect to strain; this indicates that the stress transfer in this system, between the fibre and the SWNTs, is more effective. 
Subsequent data presented in this paper will focus on samples where hot curing of the SWNT-sizing-epoxy coating has taken place. The shift rate with respect to strain has subsequently been used to convert the shift in the $2 \mathrm{D}$ band shifts recorded along a length of single fibre embedded in an epoxy resin dumbbell model composite during the fragmentation process.

\subsection{Fragmentation of the Model Composites}

To fit the stress data we used a shear lag model first proposed by Nairn [13] based on the deformation of a single fibre embedded in an infinite matrix. In Nairn's theory the fibre stress $\sigma_{\mathrm{f}}$ is given by the equation

$$
\sigma_{\mathrm{f}}=E_{\mathrm{f}} \varepsilon_{\mathrm{m}}\left[1-\frac{\cosh \beta\left(\frac{l}{2}-x\right)}{\cosh \beta \frac{l}{2}}\right]
$$

where $\varepsilon_{\mathrm{m}}$ is the matrix strain and $x$ is the distance along the fibre; $\beta$ is the shear-lag parameter defined by the equation

$$
\beta=\left\{\frac{2}{r_{1}^{2} E_{\mathrm{f}} E_{\mathrm{m}}}\left[\frac{E_{\mathrm{f}} V_{\mathrm{f}}+E_{\mathrm{m}} V_{\mathrm{m}}}{\frac{V_{\mathrm{m}}}{4 G_{\mathrm{f}}}+\frac{1}{2 G_{\mathrm{m}}}\left(\left(\frac{1}{V_{\mathrm{m}}}\right) \ln \left(\frac{1}{V_{\mathrm{f}}}\right)-1-\frac{V_{\mathrm{m}}}{2}\right)}\right]\right\}^{1 / 2}
$$

where $r_{1}$ is the fibre radius, $E_{\mathrm{f}}$ and $E_{\mathrm{m}}$ are the Young's modulus of the fibre and matrix respectively, $V_{\mathrm{f}}$ and $V_{\mathrm{m}}$ are the volume fractions of the fibre and matrix respectively, 
and $G_{\mathrm{f}}$ and $G_{\mathrm{m}}$ are the shear moduli of the fibre and matrix respectively. $G_{\mathrm{m}}$ is defined by the equation

$$
G_{\mathrm{m}}=\frac{E_{\mathrm{m}}}{2(1+v)}
$$

where $v$ is Poisson's ratio of the matrix.

Typical stress distributions along a HMCF fibre length embedded in an epoxy resin matrix model composite are shown in Figure 3. It is noted that no drift in the mean position of data are observed, suggesting that at each fixed deformation level, stress relaxation has little effect on the measurements. Up to a composite matrix strain of $0.9 \%$ the fibre remains intact with a perfect bond with the matrix. Visual evidence of fragmentation is shown in Fig. 3a and stress data obtained from the measurements of Raman band positions are shown in Fig. 3b. All data were fitted using Equation 4. At a matrix strain of $1.2 \%$ the fibre undergoes fragmentation.. Each fragment remains wellbonded to the matrix and the data along each of these can again be fitted using Equation 4. Subsequent increases in the matrix strain $(1.5 \%$ and $1.8 \%)$ leads to very pronounced fragmentation, and indeed saturation; no further increase in the number of fragments was observed beyond this point. Each set of data over the fragments is again fitted with Equation 4, although the data become evidently more "saw-toothed" indicating that debonding possibly occurs. By differentiation of Equation 4 according to the equation

$$
\tau=\frac{r_{1}}{2} \frac{\mathrm{d} \sigma_{\mathrm{f}}}{\mathrm{d} x}
$$


the interfacial shear stress $(\tau)$ can be derived. This function is continuous where bonding remains intact. At a fibre break, during fragmentation, the function becomes discontinuous. Figure 4 reports data derived from the fits of Equation 4 to the data in Figure 3.

Similar data were obtained for a LMCF embedded in an epoxy resin and coated with SWNTs to both sense the local stress and to enhance the fibre-matrix interface. These data are reported in Figure 5, showing the build-up of stress along the fibre lengths (Fig. 5a) and the interfacial shear stress (ISS) along the same fibre-matrix interface (Fig. 5b). Average fragment lengths $\left(l_{\mathrm{f}}\right)$ were determined and converted to critical fibre lengths $\left(l_{\mathrm{c}}\right)$ using Equation 2. These values and interfacial shear stresses obtained from Equation $3\left(\tau_{\mathrm{i}}\right)$, compared to the maximum values obtained using the Raman method $\left(\tau_{\max }\right)$ are reported in Table 1 for all samples. From these data a number of points are evident. It is noted that the addition of $\mathrm{COOH}$ modified SWNTs to the fibres' surfaces (for both HMCFs and LMCFs) leads to a significant increase in the interfacial shear stress. No increase is noted for the addition of HiPCO SWNTs to either fibre type. This increase in the interfacial shear stress is in agreement with our previous study [7].

The maximum interfacial shear stress $\left(\tau_{\max }\right)$ is plotted as a function of matrix strain in Figure 6 for HMCFs either in their virgin state or coated with SWNTs. The significant difference between $\mathrm{COOH}$ modified and HiPCO SWNT coated samples is clear. The reasons for the difference may be the improved interaction between $-\mathrm{OH}$ moieties in the epoxy resin and the carboxylic $(\mathrm{COOH})$ groups on the SWNTs. There is also some 
evidence that above a matrix strain of $1.8 \%$, a small decrease in the interfacial shear stress occurs. This decrease may be due to the plastic deformation, or yielding of the matrix. It has been previously reported that the shear yield stress of this particular formulation of epoxy resin is $\sim 46 \mathrm{MPa}$ [12]. All values obtained using the Raman spectroscopic method exceed this value (see Table 1). The small decrease in the $\tau_{\max }$ values may be due to an exceeding of the shear yield stress of the resin thereby causing matrix yielding. The presence of the SWNTs in the resin may also increase the shear yield strength of the resin, which could be why the $\tau_{\max }$ values exceed $46 \mathrm{MPa}$, but this remains a topic for further study. In all systems the Raman spectroscopic method of determining interfacial shear stresses returns larger values than for the traditional approach using critical fibre lengths. This may be due in part to the very high stress gradients ( $\mathrm{d} \sigma_{f} / \mathrm{d} x$ ) observed close to the ends of each fragment but also to the very different way in which the interfacial shear stress is determined using each method. Typical profiles for the shear and interfacial shear stresses are sketched for the KellyTyson and Raman methods in Figure 7. The Kelly-Tyson theory assumes a trapezoidal increase in fibre stress to a constant value in the central region, and a constant shear stress along the interface. In reality both parameters are this is a continually changing parameter with a maximum value where $\mathrm{d} \sigma_{f} / \mathrm{d} x$ is maximum. The Kelly-Tyson model therefore effectively gives an average constant value for $\tau_{\max }$, which is typically less than the value determined using the Raman method since the slopes of the sides of the trapezoids have a smaller gradient than the shear lag profile. 


\section{Conclusions}

Raman spectroscopy has been demonstrated to be a powerful tool for the analysis of carbon fibre-epoxy model composites undergoing fragmentation. For the first time it has been demonstrated that this process can be followed in a low modulus carbon fibre system provided that single wall carbon nanotubes are coated on the fibres' surface. This coating enables the following of shifts in the position of a Raman band located at $\sim 2660 \mathrm{~cm}^{-1}$ (the 2D Raman band) along a fibre length embedded in a model composite. During fragmentation the interfacial shear stress has been derived for both a high and low modulus carbon fibre systems. The use of carboxylic acid modified single wall carbon nanotubes leads to a significant increase in the interfacial shear stress; no significant increases are noted for HiPCO single wall carbon nanotubes. Some evidence that matrix yielding also takes place close to the fibre ends, where the maximum interfacial shear stress occurs, has also been presented. The presence of the functionalised nanotubes may also enhance the shear yield stress of the epoxy resin.

\section{References}

[1] Kelly A, Tyson WR. Tensile properties of fibre-reinforced metals - copper/tungsten and copper/molybdenum. Journal of the Mechanics and Physics of Solids. 1965;13(6):329-350.

[2] Harris B. Engineering composite materials. London: IOM Communications Ltd.; 1999.

[3] Harris B, Braddell OG, Lefebvre C, Verbist J. The surface-treatment of carbonfibers by electro-polymerization and plasma polymerization. Plastics Rubber and Composites Processing and Applications. 1992;18(4):221-240.

[4] Huang YL, Young RJ. Analysis of the fragmentation test for carbon-fiber epoxy model composites by means of Raman-spectroscopy. Composites Science And Technology. 1994;52(4):505-517.

[5] Montes-Moran MA, Martinez-Alonso A, Tascon JMD, Young RJ. Effects of plasma oxidation on the surface and interfacial properties of ultra-high modulus carbon fibres. Composites Part A-Applied Science And Manufacturing. 2001;32(3-4):361-371.

[6] Montes-Moran MA, Young RJ. Raman spectroscopy study of high-modulus carbon fibres: effect of plasma-treatment on the interfacial properties of single-fibre-epoxy 
composites - Part II: Characterisation of the fibre-matrix interface. Carbon.

2002;40(6):857-875.

[7] Jin S-Y, Young RJ, Eichhorn SJ. Hybrid carbon fibre - carbon nanotube composite interfaces. Composites Science and Technology. 2014;95(1):114-120.

[8] Ferrari AC, Robertson J. Interpretation of Raman spectra of disordered and amorphous carbon. Physical Review B. 2000;61(20):14095-14107.

[9] Melanitis N, Galiotis C, Tetlow PL, Davies CKL. Monitoring the micromechanics of reinforcement in carbon-fiber epoxy-resin systems. Journal of Materials Science. 1993;28(6):1648-1654.

[10] Cooper CA, Young RJ, Halsall M. Investigation into the deformation of carbon nanotubes and their composites through the use of Raman spectroscopy. Composites Part A-Applied Science And Manufacturing. 2001;32(3-4):401-411.

[11] Galiotis C, Batchelder DN. Strain dependences of the 1st-order and 2nd-order Raman-spectra of carbon-fibers. Journal of Materials Science Letters. 1988;7(5):545547.

[12] Andrews MC. Stress transfer in aramid/epoxy model composites. PhD thesis, UMIST, Manchester Materials Science Centre; 1994.

[13] Nairn JA. On the use of shear-lag methods for analysis of stress transfer unidirectional composites. Mechanics of Materials. 1997;26(2):63-80.

[14] Young RJ, Andrews MC. Deformation micromechanics in high-performance polymer fibers and composites. Materials Science and Engineering A-Structural Materials Properties Microstructure and Processing. 1994;184(2):197-205.

Table 1 Summary of the average fragment lengths $\left(l_{\mathrm{f}}\right)$, the critical lengths $\left(l_{\mathrm{c}}\right)$, the interfacial shear stresses derived using the critical lengths $\left(\tau_{\mathrm{i}}\right)$ and using the Raman spectroscopic method $\left(\tau_{\max }\right)$; HMCF - high modulus carbon fibre, LMCF - low modulus carbon fibre, $\mathrm{COOH}$ - carboxylic acid modified single wall carbon nanotubes (SWNTs). Errors are standard deviations from the mean.

\begin{tabular}{|c|c|c|c|c|}
\hline Sample & $l_{\mathrm{f}}(\mathrm{mm})$ & $l_{\mathrm{c}}(\mathrm{mm})$ & $\tau_{\mathrm{i}}(\mathrm{MPa})$ & $\tau_{\max }(\mathrm{MPa})$ \\
\hline HMCF/Epoxy & $0.40 \pm 0.01$ & $0.53 \pm 0.01$ & $36.8 \pm 0.3$ & $52.8 \pm 0.2$ \\
\hline $\begin{array}{c}\text { LMCF/Epoxy + HiPCO } \\
\text { SWNTs }\end{array}$ & $0.87 \pm 0.05$ & $1.16 \pm 0.01$ & $25.2(0.02)$ & $52.9 \pm 0.1$ \\
\hline $\begin{array}{c}\text { LMCF/Epoxy + COOH } \\
\text { SWNTs }\end{array}$ & $0.79 \pm 0.05$ & $1.05 \pm 0.01$ & $28.0(0.02)$ & $62.3 \pm 0.1$ \\
\hline $\begin{array}{c}\text { HMCF/Epoxy + HiPCO } \\
\text { SWNTs }\end{array}$ & $0.39 \pm 0.01$ & $0.52 \pm 0.01$ & $37.7 \pm 0.4$ & $53.1 \pm 0.3$ \\
\hline $\begin{array}{c}\text { HMCF/Epoxy + COOH } \\
\text { SWNTs }\end{array}$ & $0.33 \pm 0.01$ & $0.44 \pm 0.01$ & $44.6 \pm 0.3$ & $64.8 \pm 0.5$ \\
\hline
\end{tabular}


(a)

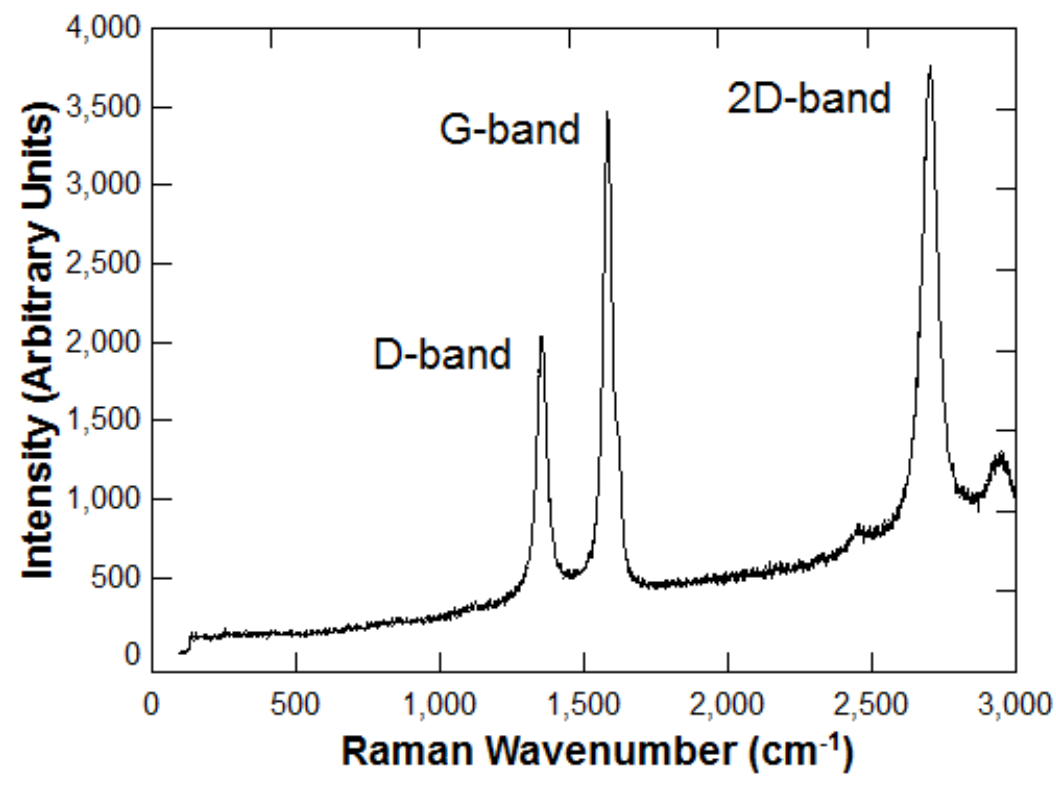

(b)

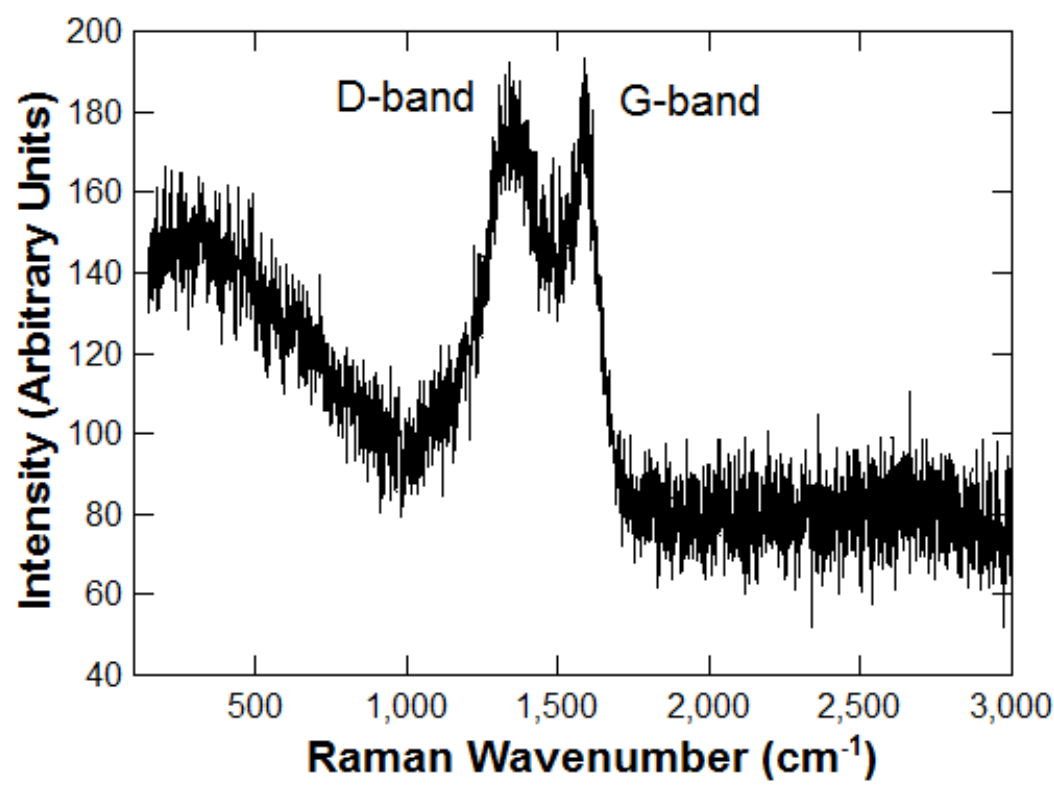


(c)

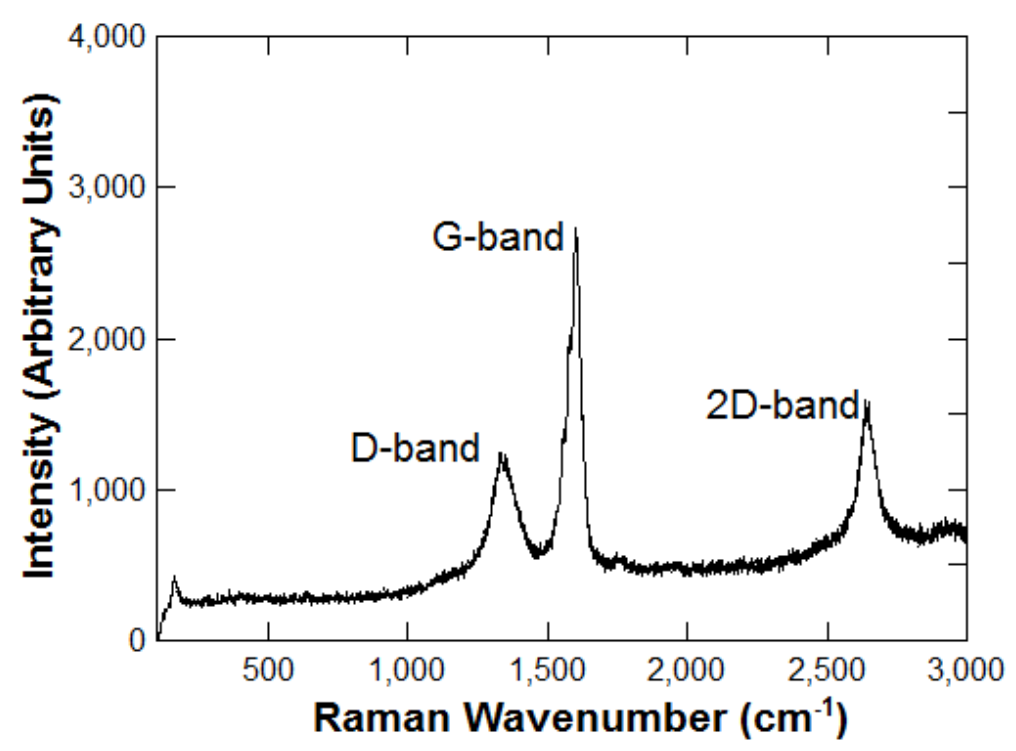

(d)

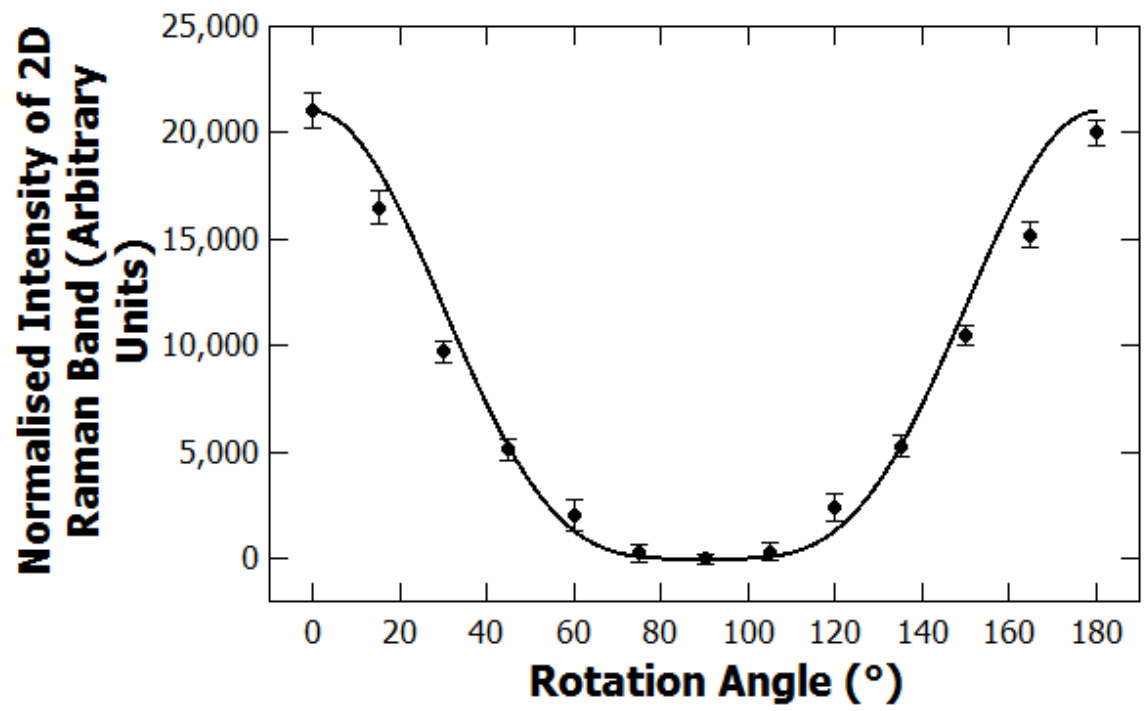

Figure 1 Typical Raman spectra for (a) a high modulus carbon fibre (HMCF), (b) a low modulus carbon fibre (LMCF), (c) single wall carbon nanotubes coated with $\mathrm{COOH}$ groups and (d) normalised intensity of the 2D Raman band as a function of the rotation angle with respect to a parallel-parallel polarisation (VV) for a low modulus carbon fibre (LMCF) coated with SWNTs. Solid line is a fit of the form $y=A+B \cos ^{4} \theta$. 
(a)

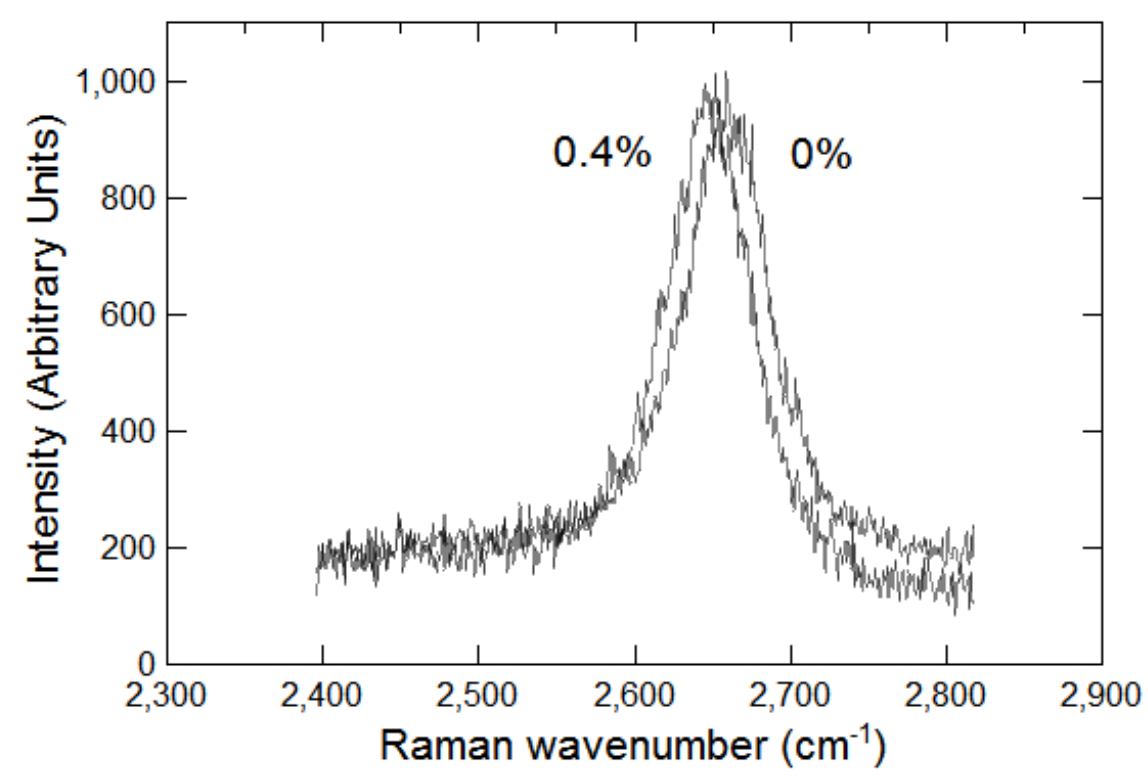

(b)

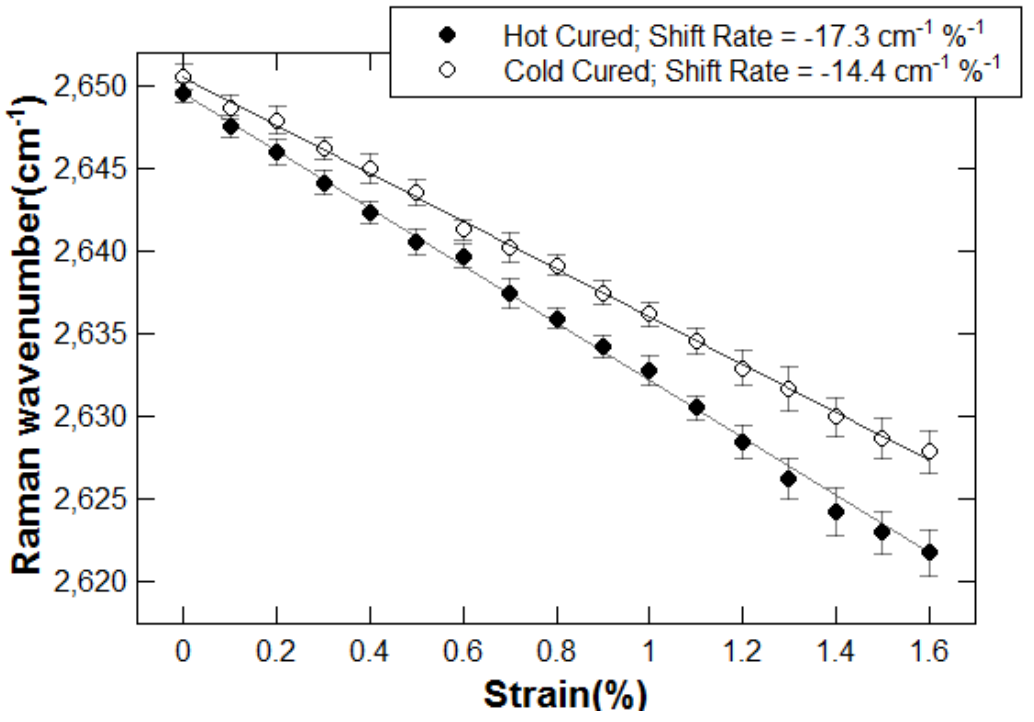

Figure 2 Typical shifts in the position of a Raman band located at $\sim 2660 \mathrm{~cm}^{-1}$ (2D) as a function of fibre strain for (a) a high modulus carbon fibre (HMCF) and (b) detailed shifts in the peak positions for a low modulus carbon fibre coated with $\mathrm{COOH}$ modified single wall carbon nanotubes. Data are shown for both hot and cold cured resin layers on fibre. Solid lines are linear regressions to the data and the fit gradients are reported as "Shift Rates" and errors are standard deviations from the mean. 
(a)

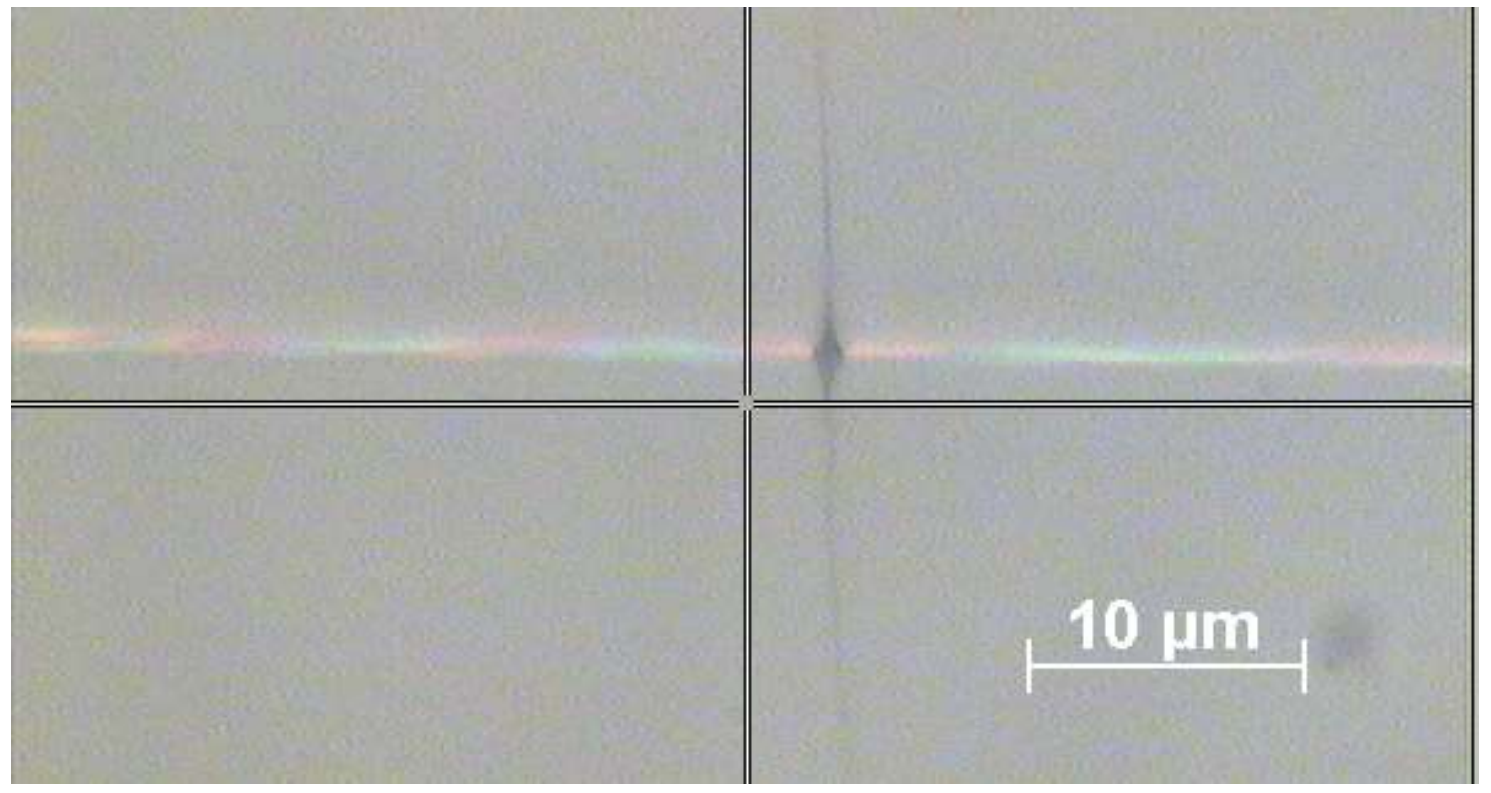

(b)

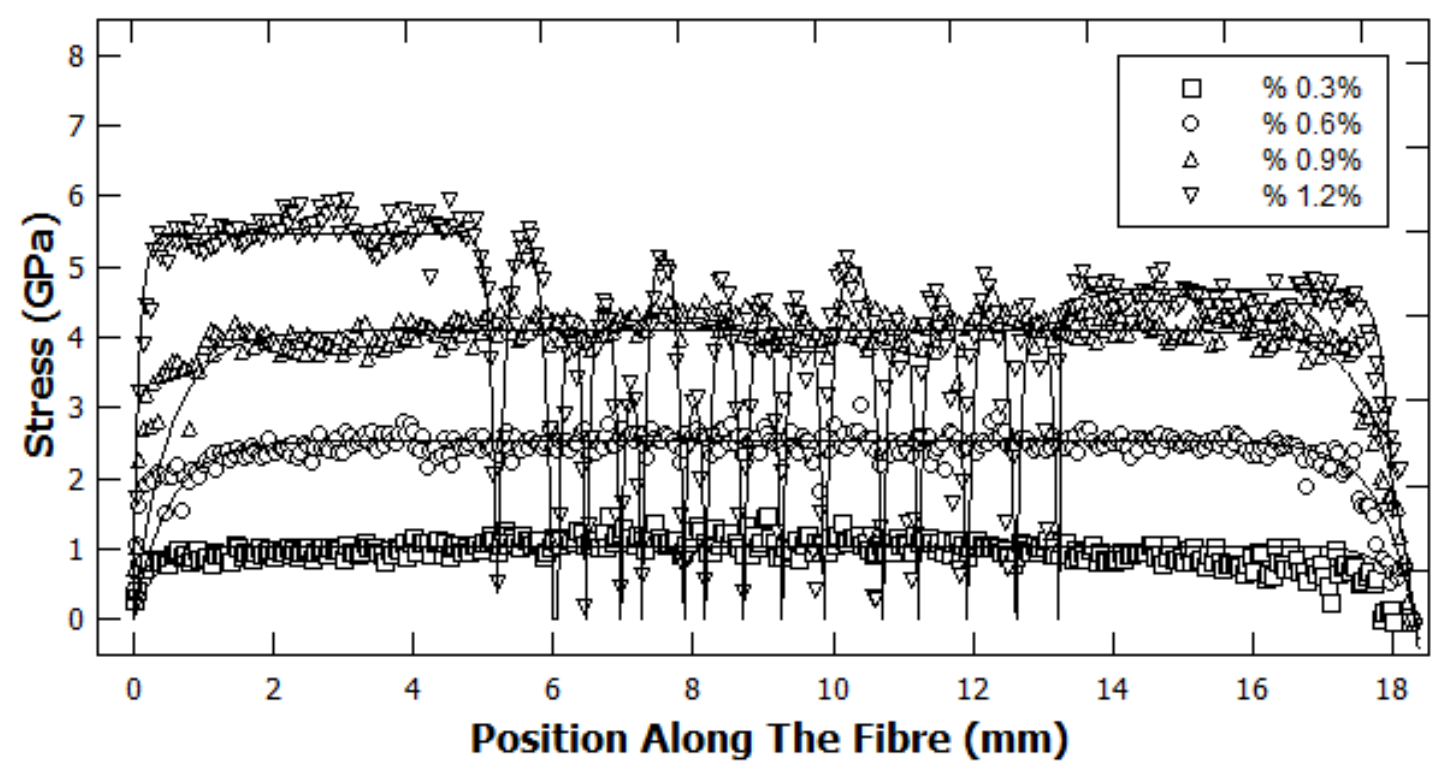


(c)

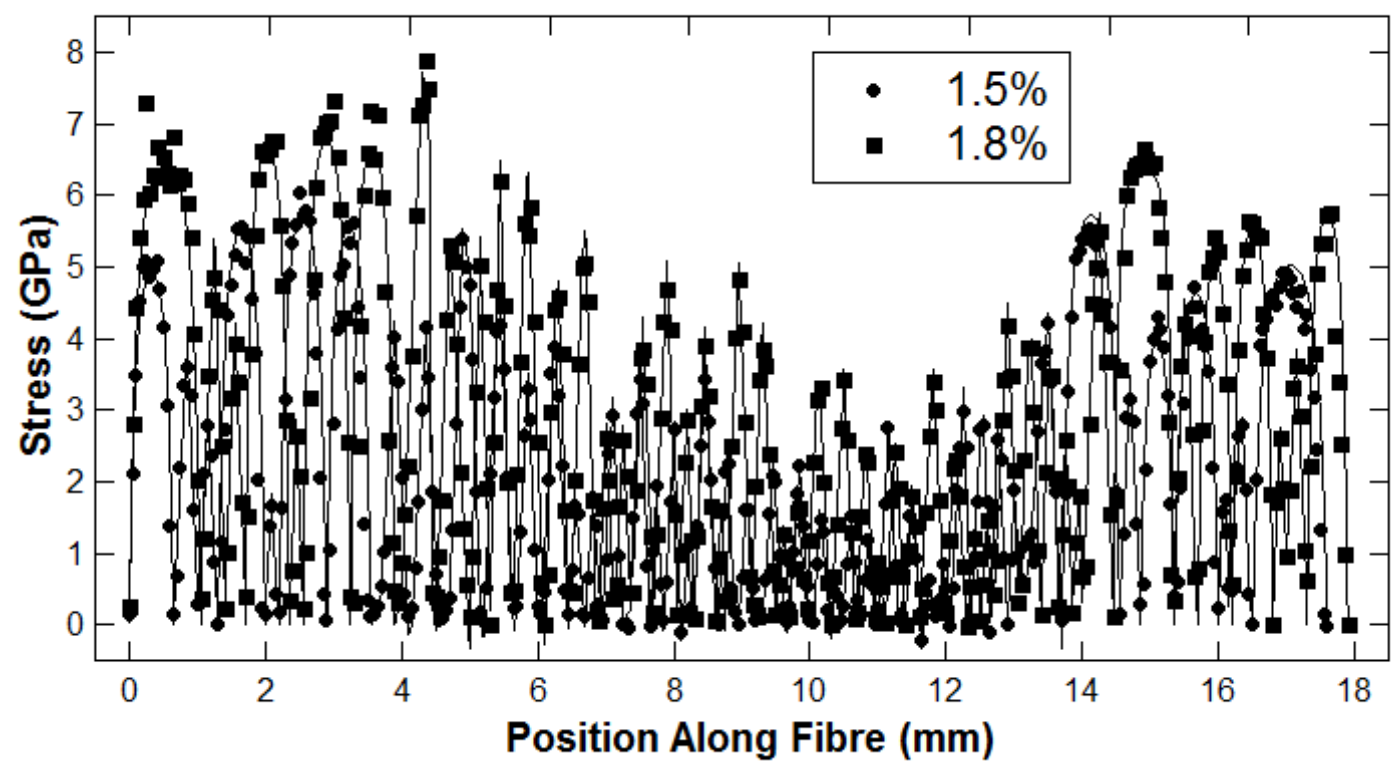

Figure 3 (a) A typical optical micrograph of a fragmented carbon fibre in a model composite. Cross-hairs are from the digital image recorded by the camera on the Raman spectrometer microscope; Typical stress distributions a single high modulus carbon fibre (HMCF) epoxy resin model composite at (b) matrix strains of $0.3-1.2 \%$ and (c) at matrix strains of 1.5 and $1.8 \%$. Solid lines are fits of Equation 4 to the data. 
(a)

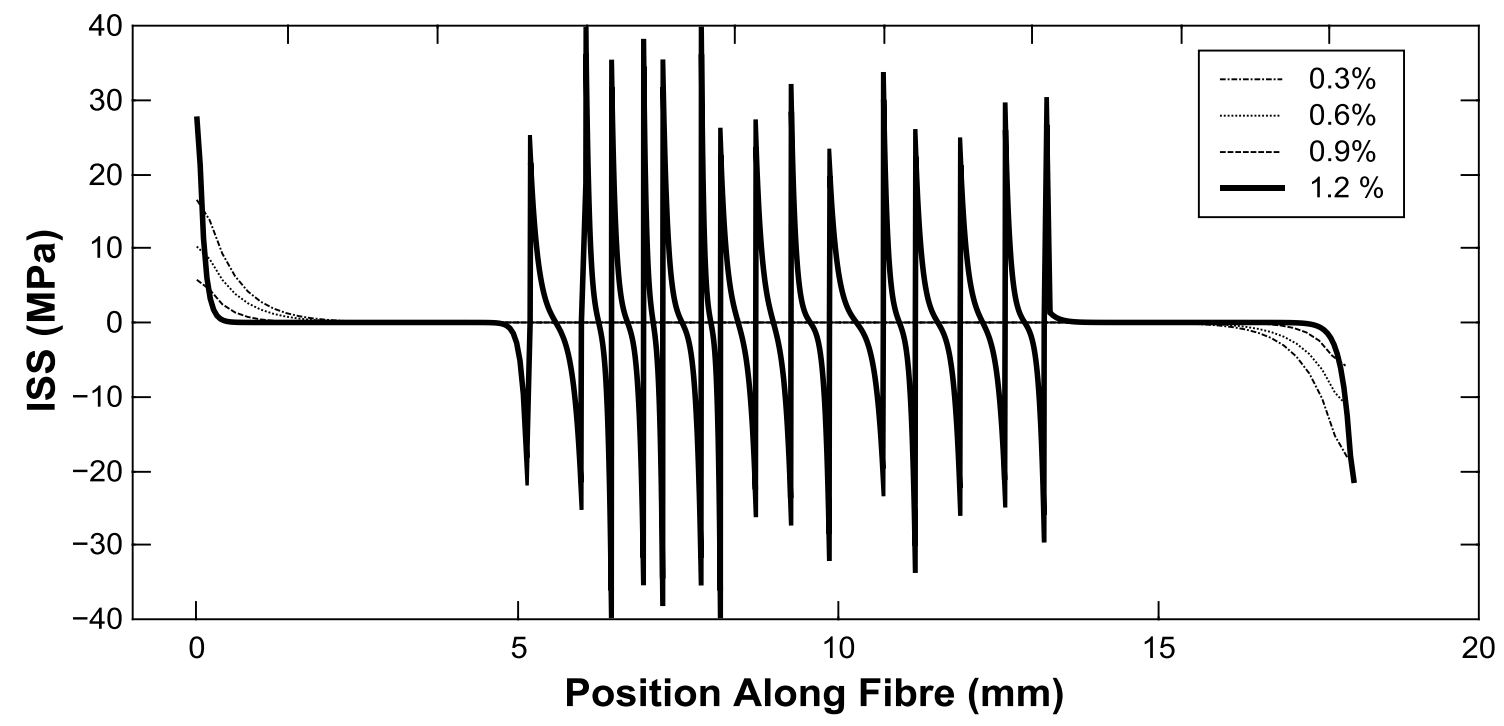

(b)

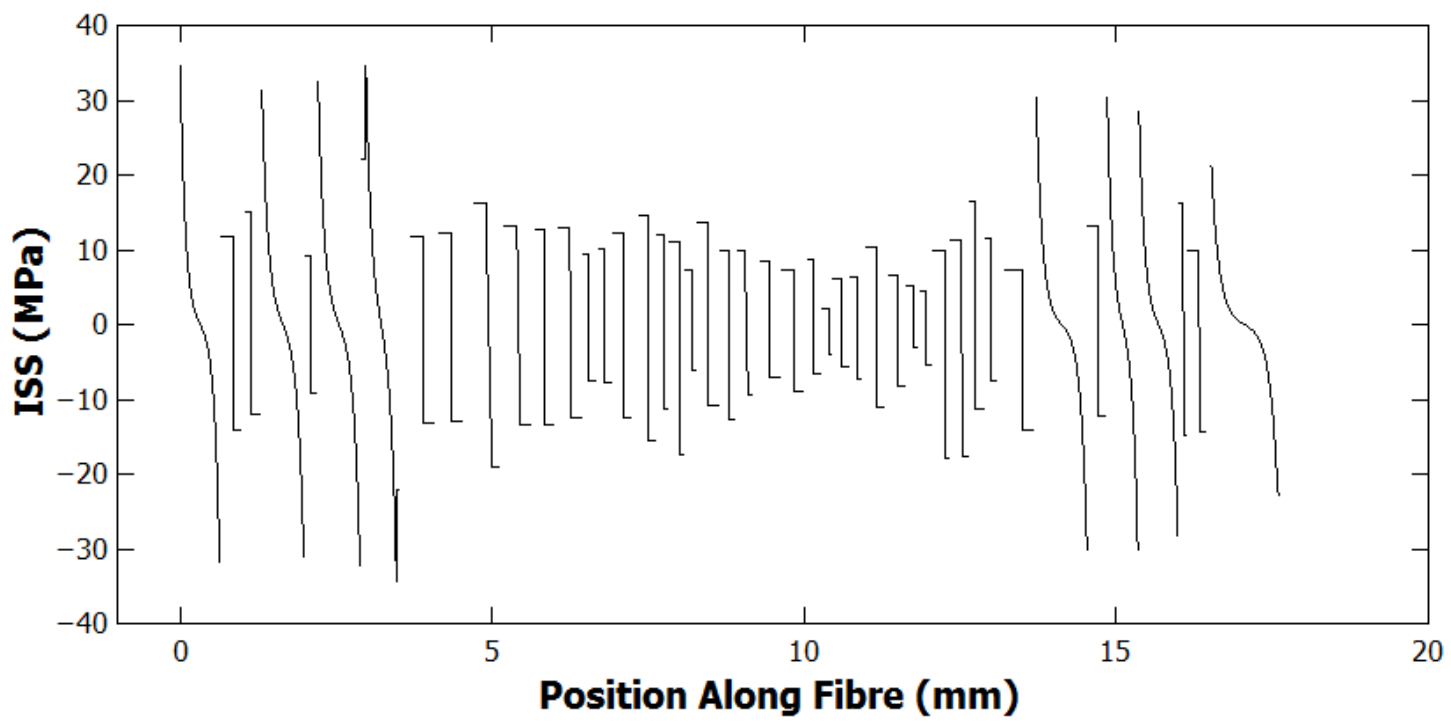

Figure 4 Interfacial shear stress (ISS) derived from Figure 3 using Equation 4 across the whole fibre-matrix interface for a high modulus carbon fibre (HMCF) epoxy resin model composite for matrix strains of (a) $0.3-1.2 \%$ and (b) $1.5 \%$ 
(a)

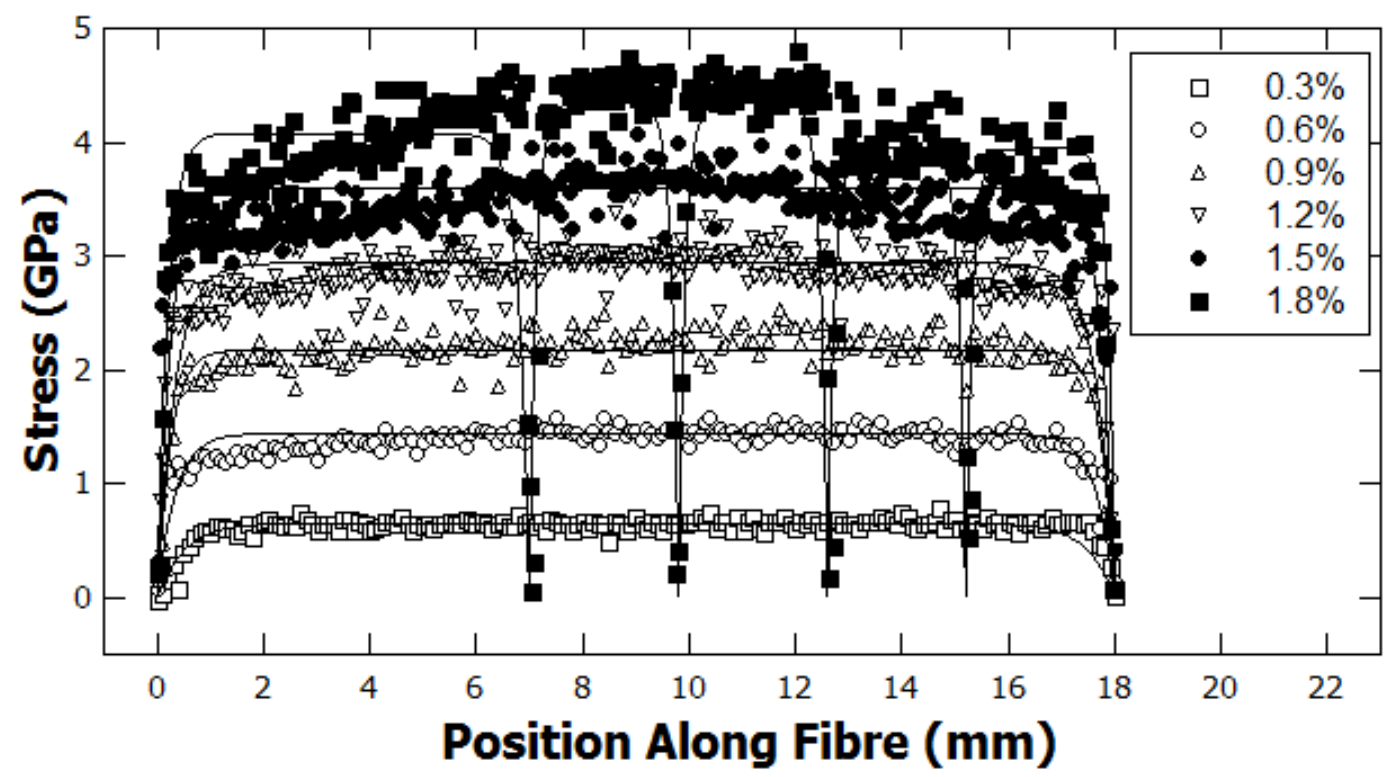

(b)

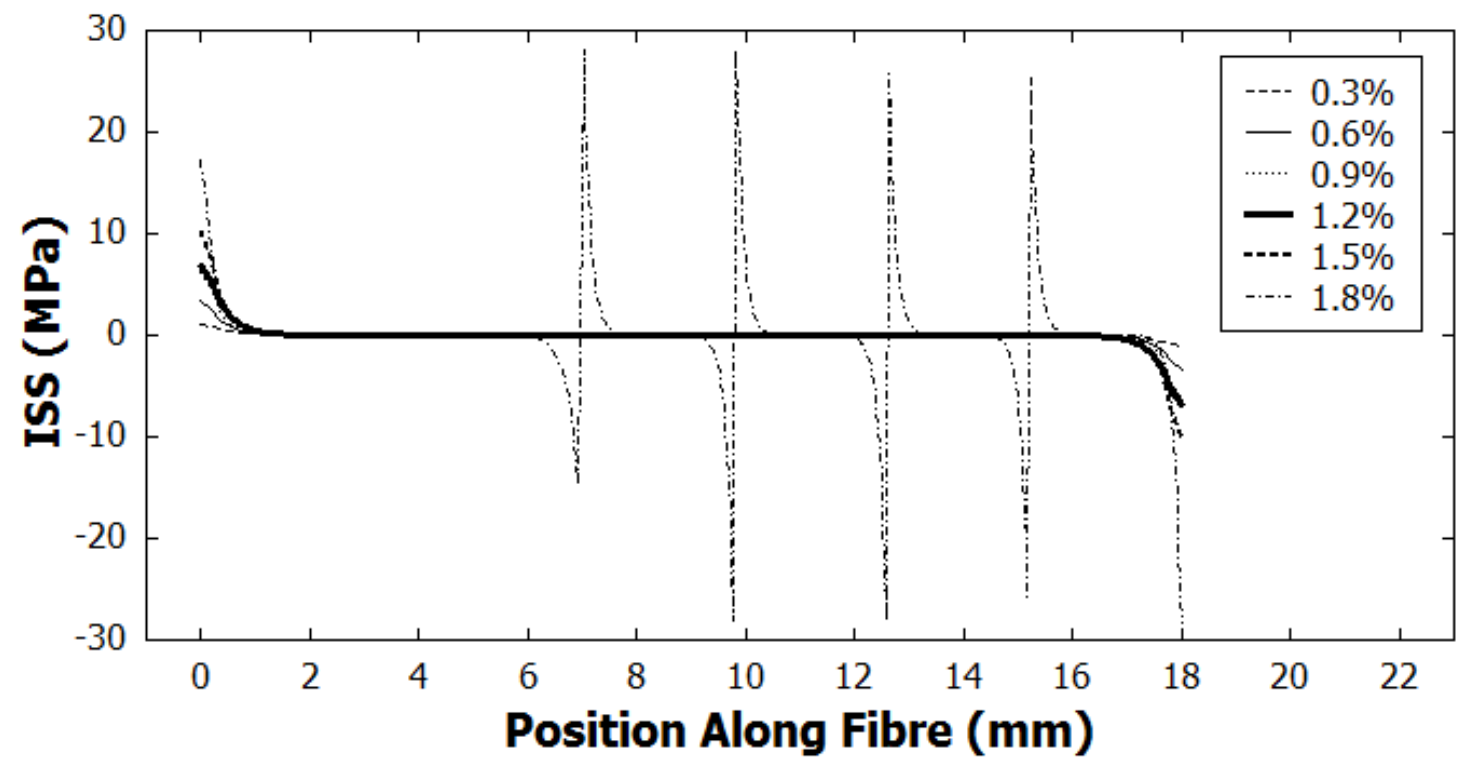

Figure 5 Typical stress distributions a single low modulus carbon fibre (HMCF) coated with HiPCO SWNTs in epoxy resin model composite at (a) matrix strains of $0.3-1.8$ $\%$ and (b) interfacial shear stress derived from (a) using Equation 4 across the whole fibre-matrix interface. 


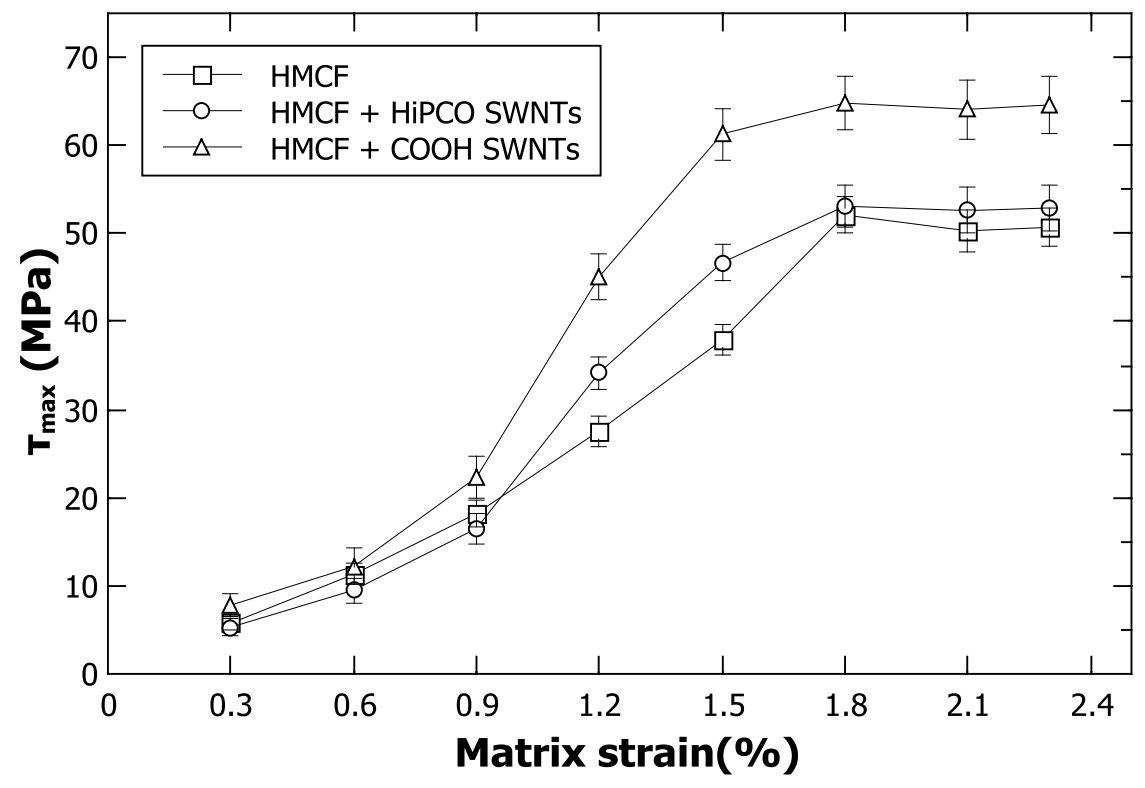

Figure 6 Interfacial shear stress as a function of matrix strain for model carbon fibreepoxy composites undergoing fragmentation; samples - High Modulus Carbon Fibres (HMCF) coated with HiPCO or carboxylated $(\mathrm{COOH})$ Single Wall Carbon Nanotubes (SWNTs). Errors are standard deviations from the mean.

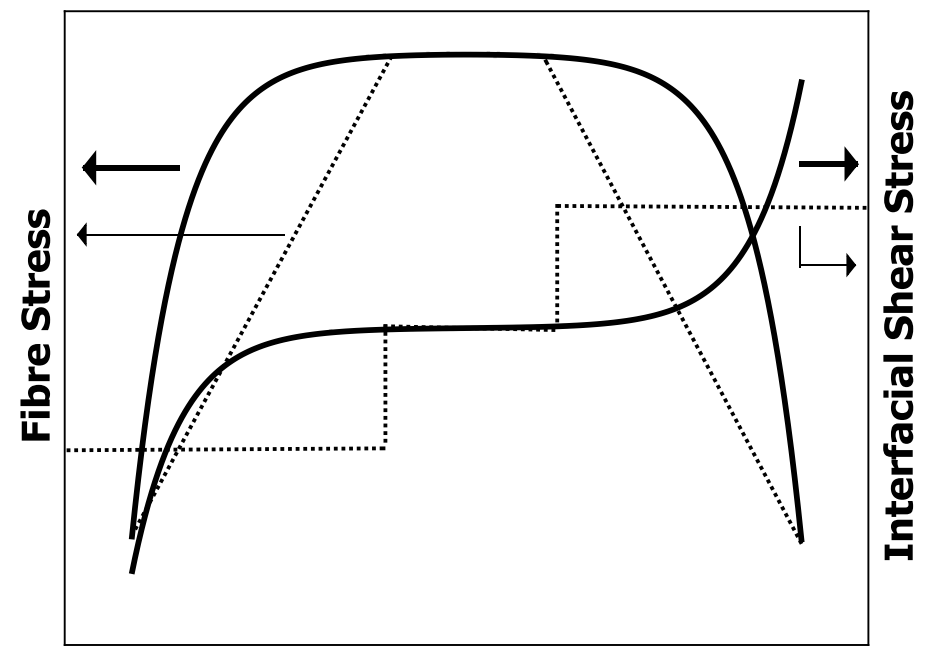

Position Along Fibre

Figure 7 Schematic of the typical shape of "Fibre Stress" and "Interfacial Shear Stress" profiles as a function of the position along a single fibre in a model composite deformed in tension as determined using Kelly-Tyson model [1] (dotted lines) and using a Raman spectroscopic method (solid lines). 